\title{
Plasmacytoid dendritic cells induce NK cell- dependent, tumor antigen-specific T cell cross-priming and tumor regression in mice
}

\author{
Chengwen Liu, ${ }^{1}$ Yanyan Lou, ${ }^{1}$ Gregory Lizée, ${ }^{1}$ Hong Qin, ${ }^{2}$ Shujuan Liu, ${ }^{1}$ Brian Rabinovich, ${ }^{1}$ \\ Grace J. Kim, ${ }^{1}$ Yi-Hong Wang, ${ }^{3}$ Yang Ye,, ${ }^{1}$ Andrew G. Sikora, ${ }^{1}$ Willem W. Overwijk, ${ }^{1}$ \\ Yong-Jun Liu, ${ }^{3}$ Gang Wang, ${ }^{1}$ and Patrick Hwu ${ }^{1}$ \\ 1Department of Melanoma Medical Oncology, ${ }^{2}$ Department of Lymphoma and Myeloma, and ${ }^{3}$ Department of Immunology, \\ Center for Cancer Immunology Research, The University of Texas MD Anderson Cancer Center, Houston, Texas, USA.
}

\begin{abstract}
A prerequisite for strong adaptive antiviral immunity is the robust initial activation of the innate immune system, which is frequently mediated by TLR-activated plasmacytoid DCs (pDCs). Natural antitumor immunity is often comparatively weak, potentially due to the lack of TLR-mediated activation signals within the tumor microenvironment. To assess whether pDCs are capable of directly facilitating effective antitumor immune responses, mice bearing established subcutaneous B16 melanoma tumors were administered TLR9-activated pDCs directly into the tumor. We found that TLR9-activated pDCs induced robust, spontaneous CTL crosspriming against multiple B16 tumor antigens, leading to the regression of both treated tumors and untreated tumors at distant contralateral sites. This $\mathrm{T}$ cell cross-priming was mediated by conventional DCs (cDCs) and was completely dependent upon the early recruitment and activation of NK cells at the tumor site. NK cell recruitment was mediated by CCR5 via chemokines secreted by $\mathrm{pDCs}$, and optimal IFN- $\gamma$ production by NK cells was mediated by OX40L expressed by $\mathrm{pDCs}$. Our data thus demonstrated that activated pDCs are capable of initiating effective and systemic antitumor immunity through the orchestration of an immune cascade involving the sequential activation of $\mathrm{NK}$ cells, $\mathrm{cDCs}$, and $\mathrm{CD8}^{+} \mathrm{T}$ cells.
\end{abstract}

\section{Introduction}

Accumulating evidence suggests that the generation of potent adaptive immunity is dependent on the initial stimulation of a strong innate immune response $(1,2)$. Pathogen exposure generates robust $\mathrm{T}$ cell-mediated immune responses largely through the recognition of pathogen-associated molecular patterns (PAMPs) by innate immune cells. Tumors by nature are not foreign and do not express PAMPs. Thus, they do not effectively activate innate immunity, and potent tumor-specific $T$ cell responses are usually not detected in cancer patients. A promising approach to improving antitumor immunity involves the application of exogenous PAMPs to boost innate immune cell activation that will subsequently trigger potent adaptive immune responses.

Many viral pathogens stimulate innate immunity through the direct activation of plasmacytoid DCs (pDCs). During viral infection, pDCs can be activated through PAMP receptors TLR7 and TLR9 to produce very high amounts of type I IFNs that, in turn, can activate monocytes, NK cells, T cells, and B cells (3-8). IFN- $\alpha$ secretion by pDCs is also known to activate conventional DCs (cDCs) to prime virus-specific T cells $(5,9)$. Thus, pDC activation early in viral infection is a crucial first step in orchestrating appropriate innate immune cell activation and the adaptive antiviral immune response that follows.

pDCs have also been implicated in the triggering of T cell-mediated autoimmunity. In patients with SLE, pDCs induce autoimmune manifestations by activating cDCs to trigger T cell responses, as well

Nonstandard abbreviations used: $\mathrm{CDC}$, conventional DC; DLN, draining LNs; i.t., intratumoral; PAMP, pathogen-associated molecular pattern; $\mathrm{PDC}$, plasmacytoid DC. Conflict of interest: The authors have declared that no conflict of interest exists. Citation for this article: J. Clin. Invest. 118:1165-1175 (2008). doi:10.1172/JCI33583. as by promoting differentiation of $\mathrm{B}$ cells into Ab-secreting plasma cells $(6,10)$. They also play a prominent role in psoriasis, where they initiate T cell-mediated immune destruction of skin cells (11).

Although pDC involvement has been clearly linked to viral immune responses and autoimmunity, it is unclear what potential impact pDCs may have in the context of initiation of antitumor immune responses. We demonstrate here within the context of an aggressive B16 mouse melanoma tumor treatment model that activated pDCs are indeed capable of initiating the orchestration of an immune cascade involving the sequential activation of $\mathrm{NK}$ cells, cDCs, and $\mathrm{CD}^{+} \mathrm{T}$ cells, ultimately culminating in effective antitumor immunity. These results provide novel insights into the mechanisms by which pDCs can bridge the gap between the innate and adaptive arms of the immune system to generate effective antitumor immune responses.

\section{Results}

Activated $p D C$ induce strong antitumor activity in vivo requiring $C D 8^{+}$ Tcells. pDCs, largely through the TLR-mediated secretion of Type I IFNs, play a critical role in initiating the generation of adaptive antiviral immune responses. To determine whether $\mathrm{pDCs}$ are capable of triggering antitumor activity in vivo, we used murine B16 melanoma, an aggressive tumor with low immunogenicity, as a tumor model. Highly purified pDCs were generated from bone marrow of mice receiving a single injection of plasmid DNA encoding Flt3L, as described previously (12). Mice were inoculated subcutaneously with B16 tumor cells, and established tumors were treated 7 days later with a single intratumoral (i.t.) injection of CpG-activated or resting pDCs.

Treatment of mice with activated but not resting $\mathrm{pDCs}$ or saline led to a significant antitumor response (Figure 1A), with concomi- 

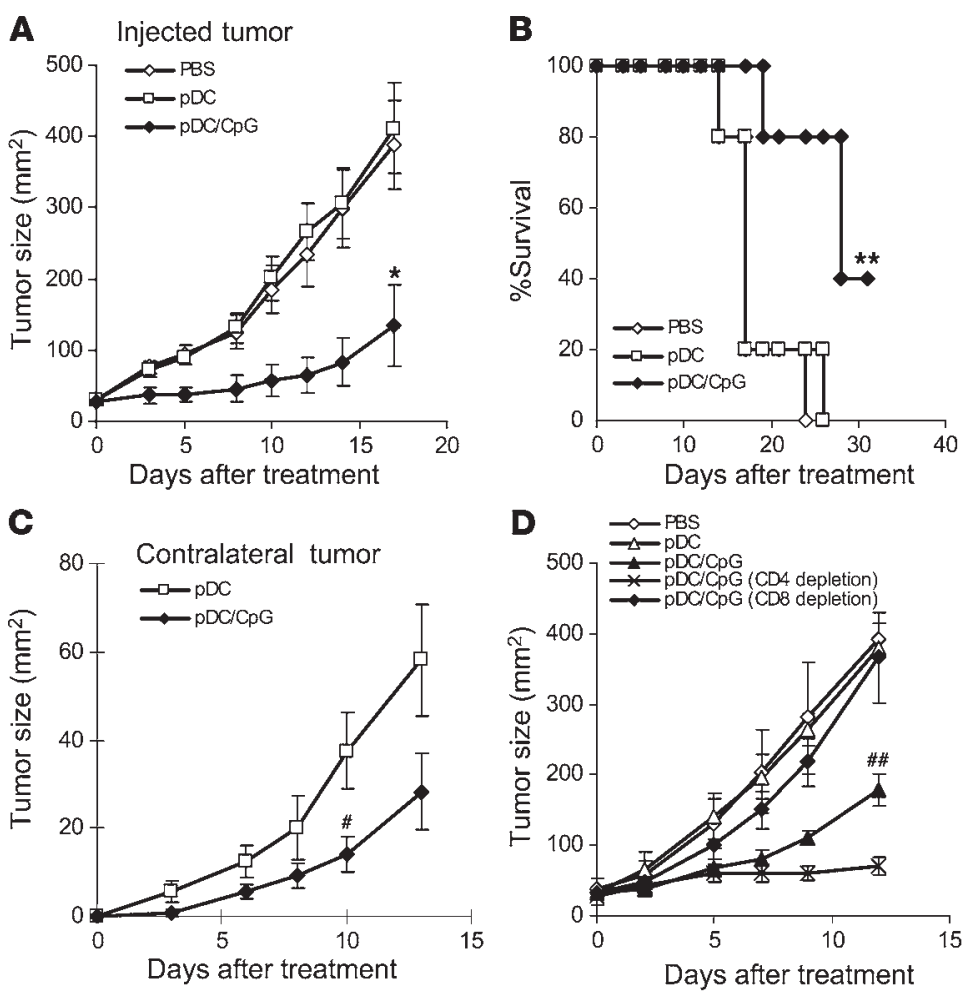

Figure 1

Activated pDC administration induces systemic antitumor activity requiring $\mathrm{CD}^{+} \mathrm{T}$ cells. Mice bearing 7-day, established subcutaneous B16 tumors were treated by i.t. injection with $\mathrm{CpG}$-activated pDCs, resting pDCs, or saline. Depicted are $(\mathbf{A})$ tumor growth $\left({ }^{*} P=0.043\right.$ for $\mathrm{pDC} / \mathrm{CpG}$ versus $\left.\mathrm{pDC}\right)$ and (B) mouse survival as monitored over time following treatment $\left({ }^{* *} P=0.02\right.$ for $\mathrm{pDC} / \mathrm{CpG}$ versus $\left.\mathrm{pDC}\right)$. (C) Mice bearing subcutaneous $\mathrm{B} 16$ tumors in both flanks were treated by i.t. pDC injection in only 1 tumor. Graph depicts growth of untreated, contralateral tumors over time following treatment with CpG-activated or resting pDCs ( $P=0.021$ for $\mathrm{pDC} / \mathrm{CpG}$ versus $\mathrm{pDC}$ ). (D) Mice bearing single subcutaneous B16 tumors were treated as described in the setting of Ab-mediated $\mathrm{CD}_{4}^{+} \mathrm{T}$ cell or $\mathrm{CD} 8^{+} \mathrm{T}$ cell depletion. Graph depicts tumor growth over time following treatment $(\# \#=0.009$ for $\mathrm{pDC} / \mathrm{CpG}$ versus $\mathrm{pDC} / \mathrm{CpG}$ with CD8 depletion). Data shown are expressed as mean \pm SEM and are representative of 2 to 3 independent experiments with similar results. tant enhanced survival (Figure 1B). Although tumors treated with a single injection of activated pDCs continued to grow over time (eventually reaching an average size of $335 \mathrm{~mm}^{2}$ by day 27), mice injected with PBS or resting pDCs reached this size 2 weeks earlier. Notably, delayed growth of an untreated tumor at a distant contralateral site was also observed following administration of activated $\mathrm{pDCs}$, suggesting that a systemic antitumor response was generated (Figure 1C). Similar antitumor activity was also observed using a MCA205 sarcoma tumor model, demonstrating that the efficacy of pDC treatment was not limited to B16 melanoma (data not shown).

To determine whether $\mathrm{CD}^{+}$or $\mathrm{CD}^{+}$cell subsets were responsible for pDC-induced B16 tumor growth inhibition, $\mathrm{CD}^{+}$or $\mathrm{CD}^{+}$cells were depleted before and during treatment. As shown in Figure $1 \mathrm{D}$, the therapeutic effects of $\mathrm{pDC}$ were abrogated in mice depleted of $\mathrm{CD}^{+}$cells but not $\mathrm{CD} 4^{+}$cells, indicating that $\mathrm{CD}^{+}$cells were required for the observed antitumor response mediated by activated pDCs. To explore the possibility that the impaired antitumor effect was simply due to depletion of injected pDC by CD4 or CD8 depletion Abs, we compared the number of pDCs at the tumor site at different time points following CD4 or CD8 depletion. As shown in Supplemental Figure 1 (supplemental material available online with this article; doi:10.1172/JCI33583DS1), although $\mathrm{Ab}$ depletion completely depleted $\mathrm{CD} 4^{+}$or $\mathrm{CD}^{+} \mathrm{T}$ cells in peripheral blood, the injected $\mathrm{PDCs}$ at the tumor site remained at similar levels with or without depletion.

Activated $p D C$ induce robust cross-priming and tumor infiltration of antigen-specific CTLs. Five days following administration of activated $\mathrm{pDCs}$, histological analysis of treated B16 tumors showed a large degree of infiltration by $\mathrm{CD}^{+} \mathrm{T}$ cells (Figure $2 \mathrm{~A}$ ). By contrast, little $T$ cell infiltration was observed following treatment of mice with resting $\mathrm{pDCs}$ or saline, further supporting the notion that
$\mathrm{CD}^{+} \mathrm{T}$ cells were mediating the antitumor response. To determine whether tumor-infiltrating $\mathrm{T}$ cells were specific for tumor-associated antigens, mice bearing B16 tumors transfected with OVA were given activated pDC treatment. Five days later, pDC-injected tumors were analyzed for numbers of infiltrating $\mathrm{OVA}_{257-264}-\mathrm{spe}-$ cific $\mathrm{T}$ cells by tetramer staining. Strikingly, in activated $\mathrm{pDC}$-treated tumors, OVA tetramer-positive cells comprised more than $40 \%$ of the total tumor-infiltrating lymphocytes (Figure 2B). Notably, in untreated contralateral tumors, $\mathrm{OVA}_{257-264-\text { specific cells still }}$ made up greater than $10 \%$ of total tumor-infiltrating lymphocytes. By contrast, treatment with resting $\mathrm{pDCs}$ or saline induced very few tumor antigen-specific CTLs (Figure 2B). Collectively, these results demonstrate that activated $\mathrm{pDC}$ administration can induce robust cross-priming of tumor antigen-specific $\mathrm{T}$ cells, leading to systemic infiltration of both treated and distant tumor sites.

The functional capacity of cross-primed $\mathrm{CD}^{+} \mathrm{T}$ cells was also examined by stimulating splenocytes harvested from B16-OVA tumor-bearing mice at treatment day 5 . These splenocytes were restimulated with or without $\mathrm{OVA}_{257-264}$ peptide, and IFN- $\gamma$ production was quantitated by intracellular staining. As shown in Figure $2 \mathrm{C}$, administration of activated $\mathrm{pDCs}$ led to over 60 -fold more functional $\mathrm{OVA}_{257-264}$-specific $\mathrm{T}$ cells compared with treatment with resting $\mathrm{pDCs}$. Although $\mathrm{pDCs}$ were extensively washed before injection, we next sought to eliminate the possibility that contaminating $\mathrm{CpG}$ was responsible for the induction of OVA-specific CTL response in our study. To this end, we injected mice i.t. with $1 \mu \mathrm{g}$ of CpG (a 50-fold higher dose than the possible maximum contaminated $\mathrm{CpG}$ in our procedure) or CpG-preincubated NK cells. As shown in Supplemental Figure 2, only mice receiving CpG-activated pDCs showed significantly increased OVA-specific CTL responses as compared with unstimulated pDCs. By contrast, mice receiving the same number of CpG-preincubated NK cells or direct injection 
A
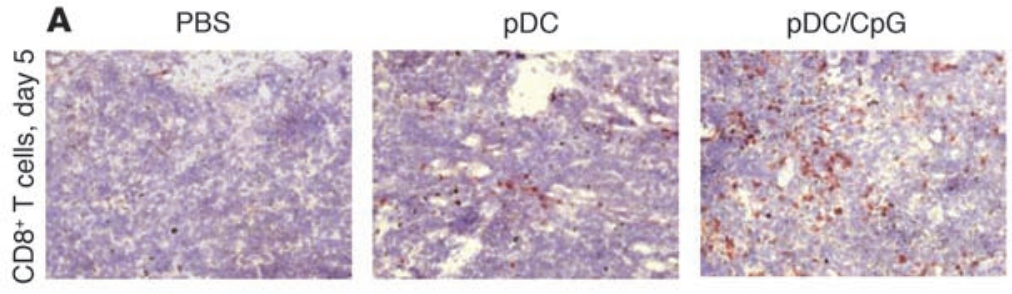

B
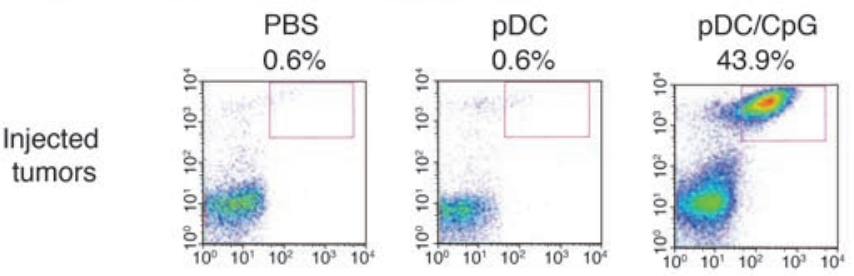
Noninjected contralateral tumors
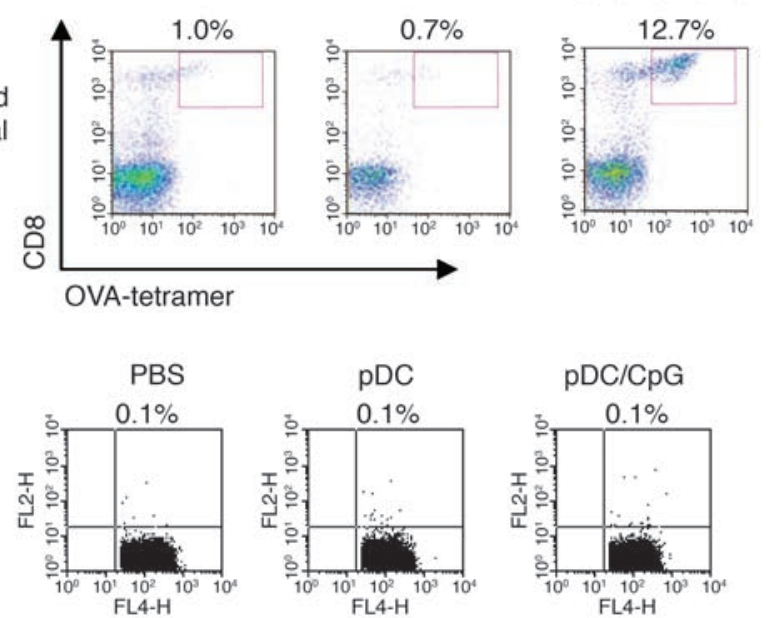

C No peptide
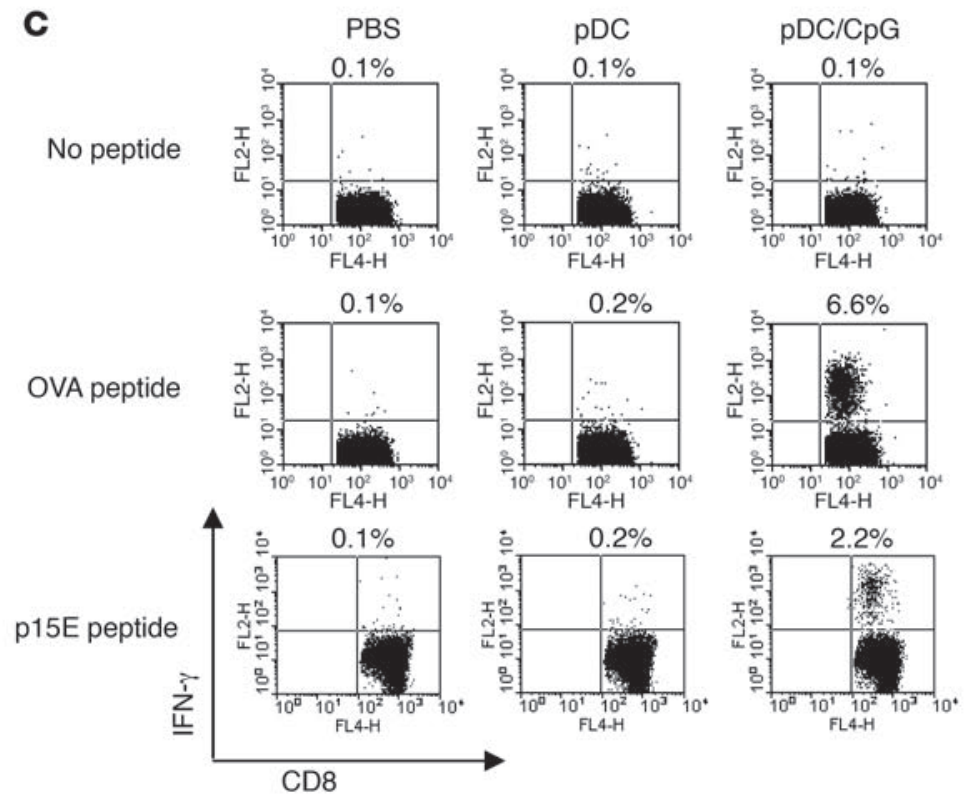

\section{Figure 2}

Activated pDCs induce robust cross-priming and tumor infiltration of antigen-specific CTLs. B16-OVA or B16 tumor-bearing mice were given i.t. pDC or saline injection. (A) Five days later tumor sections were stained for CD8 ${ }^{+} \mathrm{T}$ cells. Original magnification, $\times 100$. (B) OVAspecific $T$ cell infiltration of pDC-injected and uninjected tumors, as determined by OVA-tetramer staining. (C) Flow cytometric analysis of intracellular IFN- $\gamma$ production by splenic $C D 8^{+} \mathrm{T}$ cells with no peptide, OVA peptide, or p15E peptide restimulation.

by B16 as well as several other murine tumors (Figure 2C) (13). Thus, these data demonstrate that activated pDC treatment can induce cross-priming of multiple CTL specificities, not only against neoantigens like OVA, but also against natural, tumor self antigens.

Activated tumor antigen-positive $c D C s$ mediate $T$ cell cross-priming in tumor DLNs following $P D C$ administration. Although pDCs have recently been shown to be capable of processing and presenting exogenous antigens in vitro (14), studies performed in vivo have shown activated pDCs were capable of priming $\mathrm{CD} 8^{+} \mathrm{T}$ cell responses against endogenous but not exogenous antigens (15). We next set out to determine which type of APC was responsible for cross-priming tumor antigenspecific $T$ cells in our model. A dynamic analysis of the $\mathrm{T}$ cell response over time following i.t. $\mathrm{pDC}$ administration showed that OVA-specific T cells initially appeared in the tumor draining LNs (DLN) at day 3 to day 4, followed approximately 1 to 2 days later by $\mathrm{T}$ cell infiltration of spleen and tumor (data not shown).

To determine whether $\mathrm{pDCs}$ or endogenous cDCs were responsible for cross-priming $\mathrm{T}$ cells in tumor DLN, tumor-bearing mice were treated with $\mathrm{pDCs}$, and DLN and non-DLN were harvested 16 to 32 hours after injection. As shown in Figure 3A, tumor DLN from mice receiving activated pDCs were dramatically increased in size compared with those from mice receiving resting $\mathrm{pDCs}$ or saline. The absolute numbers of cDCs, but not pDCs, in tumor DLN was also increased approximately 3 -fold as compared with those of control mice (Figure 3B). Furthermore, DLN isolated from activated $\mathrm{pDC}$-treated mice displayed more CD86 hi-expressing cDCs as compared with control group (Figure 3C and Supplemental Figure 3A). By contrast, the numbers and activation status of cDCs

of i.t. CpG showed relatively few OVA-specific CTLs, indicating that the vast majority of CTLs we observed in our study with activated pDCs were not induced by contaminating CpG.

Although OVA-specific $\mathrm{T}$ cell responses may accurately model tumor neoantigens, many tumor antigens are self antigens and are therefore subject to stricter immunological tolerance mechanisms. Furthermore, spontaneous cross-priming against OVA is relatively robust and may not be representative of most tumor antigens. To assess whether $\mathrm{pDC}$-induced cross-priming could also be generated to other natural tumor antigens, we repeated the cross-priming study using native B16 tumors. In this setting, we found that activated pDCs could induce a strong CTL response against a natural, endogenous retroviral envelope protein, p15E, expressed and $\mathrm{pDCs}$ remained unchanged in non-DLN (data not shown).

To examine whether $\mathrm{pDCs}$ or endogenous $\mathrm{cDCs}$ were responsible for directly inducing $\mathrm{T}$ cell cross-priming in DLN through the uptake and cross-presentation of tumor-associated antigens, we repeated the experiment using B16 tumors transduced to express both OVA and GFP. B16-OVA-GFP tumor-bearing mice were given pDC treatment, and tumor DLN and non-DLN were isolated 16 to 32 hours later. As assessed by flow cytometry, DLN of mice injected with activated $\mathrm{pDCs}$ contained more $\mathrm{GFP}^{+} \mathrm{cDCs}$ compared with mice treated with resting pDCs or saline (Figure 3D and Supplemental Figure 3B). By contrast, pDCs isolated from tumor DLN contained no detectable GFP. To determine which APCs isolated from tumor DLN were capable of activating OVA-specific 

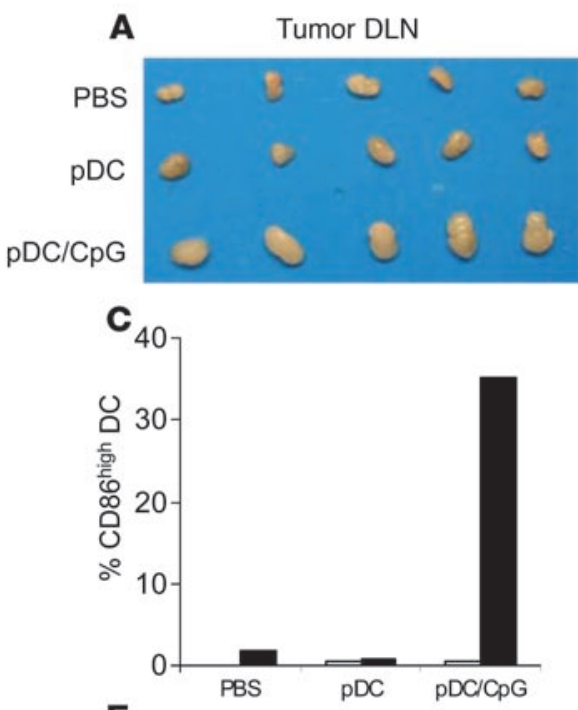

E

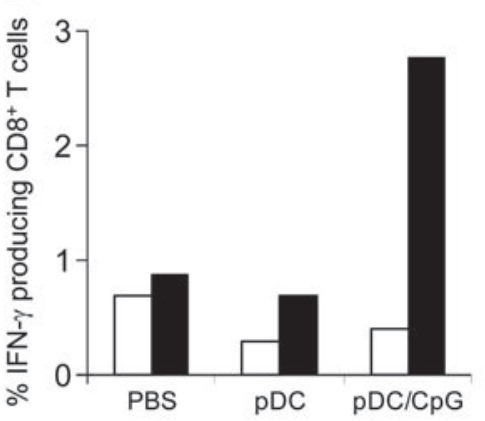

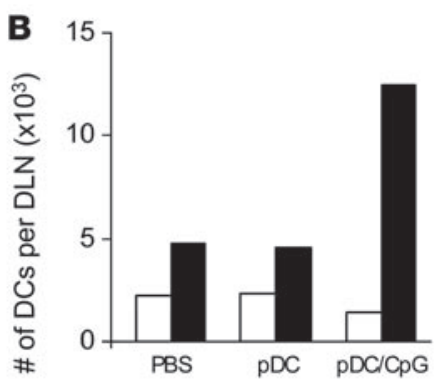

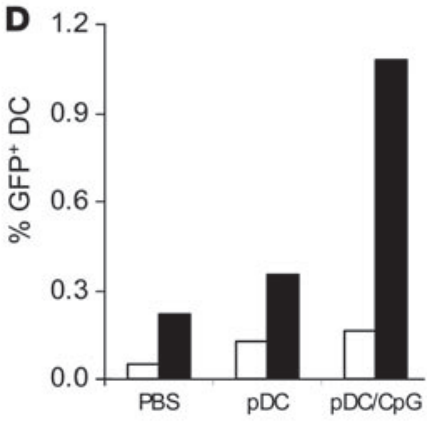

$\square \mathrm{pDC}$

- $\mathrm{CDC}$

\section{Figure 3}

pDC treatment enhances cDC activation, tumor antigen uptake, cross-presentation, and migration to tumor DLN. B16-OVA or B16-OVA-GFP tumorbearing mice (5 to 6 mice per group) were injected i.t. with resting pDCs, CpG-activated pDCs, or saline, and 16 to 32 hours later tumor DLN were harvested. (A) Photograph showing tumor DLN of treated mice. (B) Graph depicting absolute numbers of cDCs and pDCs per DLN. (C) Activation status of cDCs and pDCs, as determined by the percentage of CD86 hi DCs. (D) Percentages of tumor-associated antigen-positive (GFP+) CDCs and pDCs in tumor DLN. (E) OVA cross-presentation by DLNderived $\mathrm{cDCs}$ and $\mathrm{pDCs}$, as determined by activation of IFN- $\gamma$ secretion by OT-I T cells. Data are representative of 3 independent experiments with similar results.

during $\mathrm{pDC}$ treatment. As shown in Figure 4C, mice receiving NK cell depletion demonstrated a dramatic reduction in tumor-infiltrating $\mathrm{CD}^{+} \mathrm{T}$ cells, suggesting that NK cells were crucial for mediating the $\mathrm{pDC}$-induced $\mathrm{CD}^{+}$ $\mathrm{T}$ cell responses. Supporting this notion, NK cell depletion also completely abrogated crosspriming of functional OVA-specific and p15specific T cells (Figure 4D). Furthermore, pDC treatment was rendered completely ineffective in inhibiting tumor growth when NK cells were depleted, demonstrating that the antitumor immune response was dependent on NK cells (Figure 4E).

T cells, we sorted cDC (CD11 $\left.\mathrm{c}^{+} \mathrm{B} 220^{-} \mathrm{DX} 5^{-} \mathrm{CD} 19^{-} \mathrm{CD} 3^{-}\right)$and $\mathrm{pDC}$ $\left(\mathrm{CD} 11 \mathrm{c}^{\left.\mathrm{int} B 220^{+} \mathrm{DX} 5^{-} \mathrm{CD} 19^{-} \mathrm{CD} 3^{-}\right)}\right.$populations and separately cocultured these cells with OVA-specific OT-I T cells. As shown in Figure 3E, only ex vivo-isolated cDCs, but not $\mathrm{pDCs}$, induced IFN- $\gamma$ production by OT-I T cells. These results suggested that $\mathrm{cDCs}$ were responsible for acquiring and processing tumor-associated antigens and cross-priming tumor antigen-specific T cells.

Early NK cell tumor infiltration is critical for $p D C$-mediated $T$ cell cross-priming and antitumor activity. The presence of tumor antigens within $\mathrm{cDCs}$ isolated from tumor DLN and generation of tumor antigen-specific CTLs implied that early tumor cell lysis had occurred, liberating antigens for uptake by APCs. As IFN- $\alpha$ is known to activate NK cell cytotoxicity $(5,16)$, we next examined whether NK cells played a role in tumor cell lysis at early time points in our pDC treatment model. Histological analysis of B16 tumors following administration of activated pDCs showed a dramatic infiltration of NK cells by day 2 (Figure 4A). By contrast, no such infiltration was seen following treatment of mice with nonactivated pDCs or saline. Along with increased NK cell numbers, tumor-infiltrating NK cells also demonstrated a more activated phenotype, as assessed by expression of the activation marker CD69 (Figure 4B). This activation of tumor-infiltrating NK cells by activated pDCs was not impaired by CD4 or CD8 depletion, further confirming that the $\mathrm{Ab}$ depletion likely had a minimal impact on injected pDCs (Supplemental Figure 4).

To determine whether tumor infiltration by activated NK cells was important for initiating subsequent $\mathrm{T}$ cell responses and antitumor activity, mice were depleted of NK cells prior to and
NK cell recruitment is driven by CCR5 chemokine ligands produced by activated $p D C s$. The rapid influx of NK cells into tumor tissues following injection of activated $\mathrm{pDCs}$ suggested that $\mathrm{pDCs}$ may directly induce NK cell accumulation at the tumor site. To explore this hypothesis further, established B16 tumors were harvested 2 days following pDC injection and analyzed by flow cytometry. As shown in Figure 5A, injection of activated pDCs increased the percentage of NK cells within the tumor microenvironment approximately 5- to 10 -fold compared with mice injected with resting $\mathrm{pDCs}$ or saline. To determine whether increased NK cell accumulation was due to enhanced proliferation at the tumor site, an in situ BrdU incorporation experiment was performed. These experiments, however, could not detect significant proliferation of NK cells within the tumor microenvironment, indicating that NK cell accumulation in the tumors was likely not due to in situ NK cell proliferation (data not shown).

To further dissect the mechanism of NK cell i.t. accumulation, we next examined whether enhanced NK cell chemotaxis to tumor sites was induced by activated pDCs. To this end, we evaluated chemokine gene transcription by activated $\mathrm{pDCs}$ and the corresponding chemokine receptor genes expressed by NK cells using microarray analyses. Among a large panel of chemokines tested we found that, compared with resting pDCs, CpG-activated pDCs expressed high levels of chemokines CCL3, CCL4, and CCL5 as well as, to a lesser extent, CXCL10 (Figure 5B). Expression of these chemokines was also validated by measurement of chemokine protein levels in cell supernatants from activated pDCs. Consistent with the microarray results, substantial amounts of CCL3, 
A

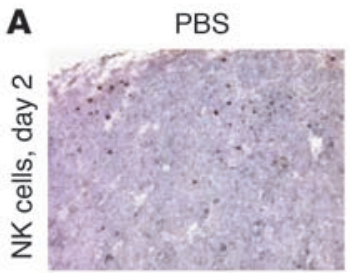

B
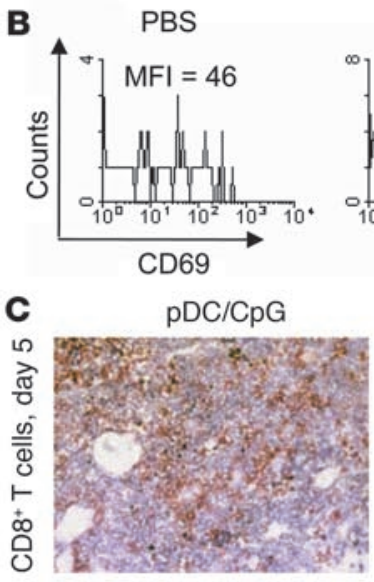

D
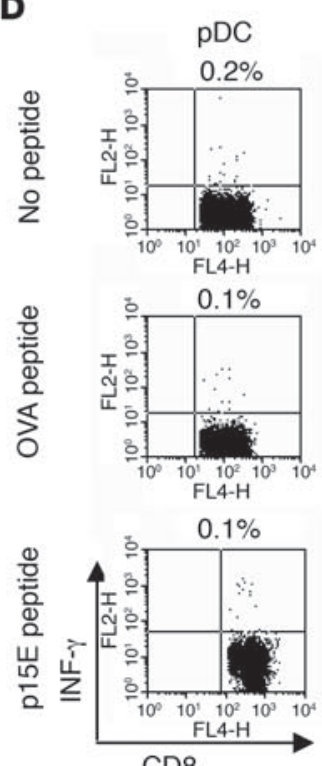

CD8
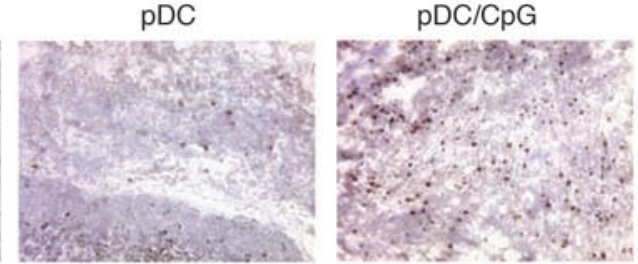

pDC
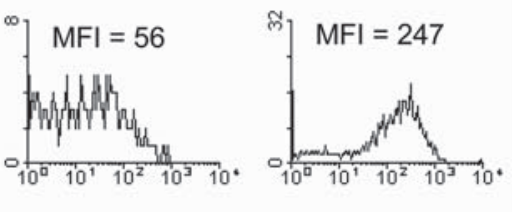

pDC/CpG (NK depletion)

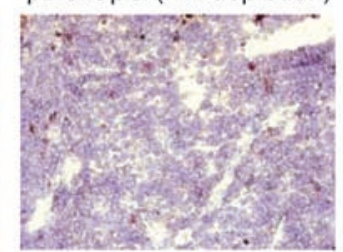

$\mathrm{pDC} / \mathrm{CpG}$

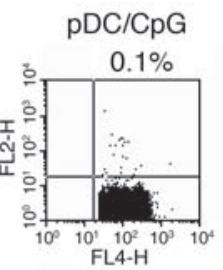

(NK depletion)
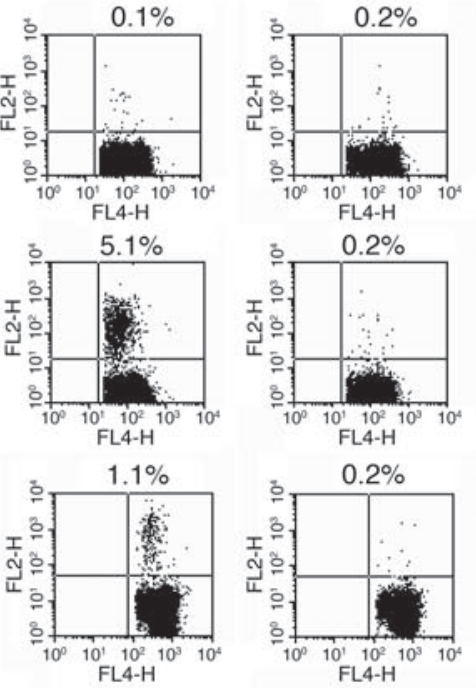

E

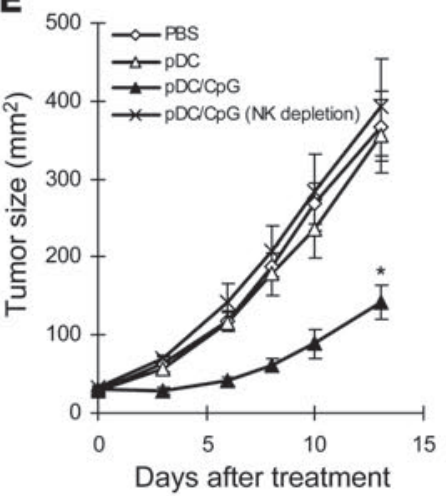

Figure 4

pDC-induced cross-priming of CTLs is dependent on NK infiltration and activation in tumors. Tumor-bearing mice were given i.t. injection of $\mathrm{CpG}$-activated $\mathrm{pDCs}$, resting $\mathrm{pDCs}$, or saline. (A) NK cell tumor infiltration at treatment day 2, as determined by immunohistochemistry. (B) Expression of CD69 on tumor-infiltrating NK cells. (C) Tumor infiltration by $\mathrm{CD}^{+} \mathrm{T}$ cells at treatment day 5 in mice receiving NK depletion as compared with controls. Original magnification, $\times 100$. (D) Flow cytometric analysis of intracellular IFN- $\gamma$ production by splenic $\mathrm{CD}^{+}$ $T$ cells with no peptide, OVA peptide, or p15E peptide restimulation, with or without NK cell depletion. (E) Mice bearing single subcutaneous B16 tumors were treated with $\mathrm{pDCs}$ or saline control. If indicated, mice were depleted of NK cells with $\mathrm{Ab}\left({ }^{*} P=0.027\right.$ for $\mathrm{pDC} / \mathrm{CpG}$ versus $\mathrm{pDC} / \mathrm{CpG}$ with NK depletion). Data shown are expressed as mean \pm SEM and are representative of 3 independent experiments with similar results.
CCL4, and CCL5 proteins were detectable in supernatants of CpG-activated pDCs (Figure 5C).

Since CCL3, CCL4, and CCL5 all bind to the chemokine receptor CCR5, we next examined whether NK cell migration into pDC-treated tumors was reduced in CCR5 ${ }^{-/}$mice. B16-bearing CCR5 $/-$ or wild-type mice were treated with activated pDCs, and the frequencies of tumor-infiltrating NK cells were evaluated. As shown in Figure 5D, CCR5 ${ }^{-/}$mice showed approximately 4-fold fewer tumor-infiltrating NK cells compared with wild-type mice, suggesting that CCR5 likely plays a key role in pDC-mediated chemotaxis of NK cells into tumors.

Activated $p D C$ stimulate NK cell cytolytic function through cytokinemediated mechanisms. Since NK cells recruited to pDC-injected tumors displayed an activated phenotype, we next explored whether
pDCs could activate NK cell effector function directly, and if so, what mechanisms were involved. To investigate this, we first examined the ability of pDCs to induce NK cell cytolytic activity in vitro. Coculture of NK cells with activated pDCs, but not resting pDCs induced strong NK cell cytotoxic activity against both YAC-1 and B16 tumor cell targets (Figure 6, A and B). Addition of mAbs specific for IFN- $\alpha / \beta$ but not of IL-12 or TNF- $\alpha$ abolished the ability of pDCs to stimulate NK cell cytotoxicity (Figure 6C). Moreover, addition of exogenous IFN- $\alpha / \beta$ to NK cultures mimicked the effects of activated pDCs, and separation of pDCs and NK cells by a transwell membrane did not diminish the cytolytic activity (Figure 6C). This finding was also confirmed in vivo, where NK cells exposed to activated pDCs demonstrated increased cytolytic activity (Figure 6D). Taken together, these data suggest that type I 
A

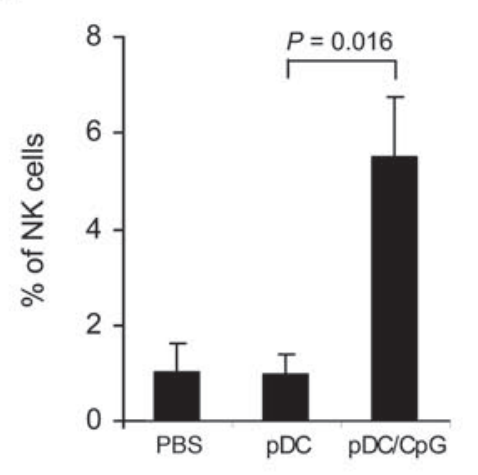

B

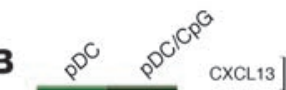

D.

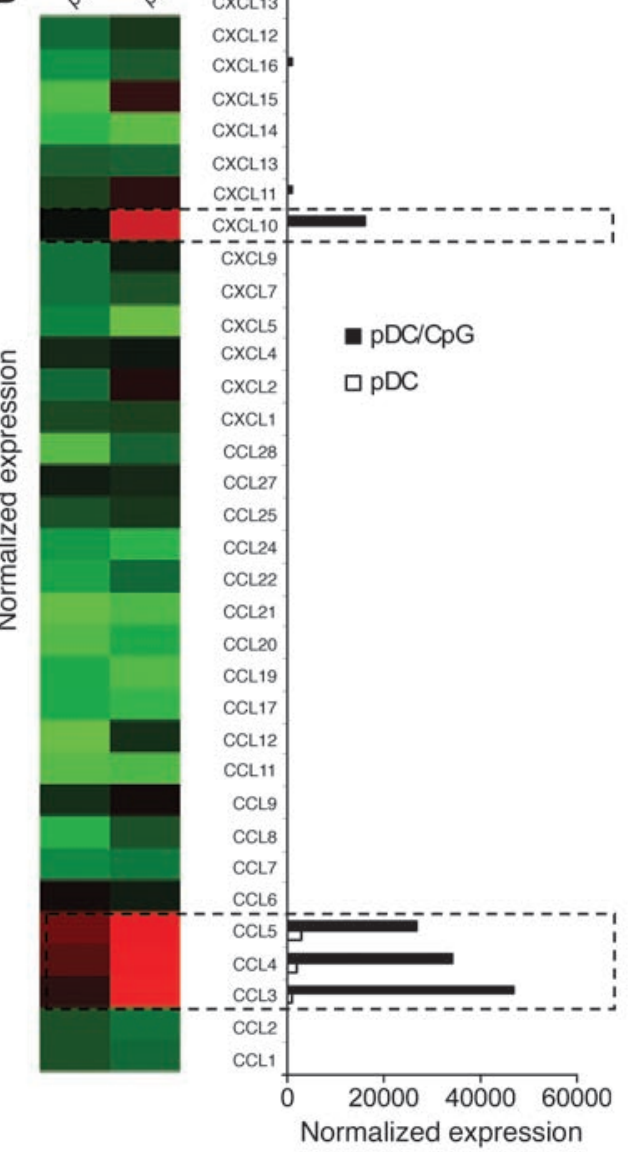

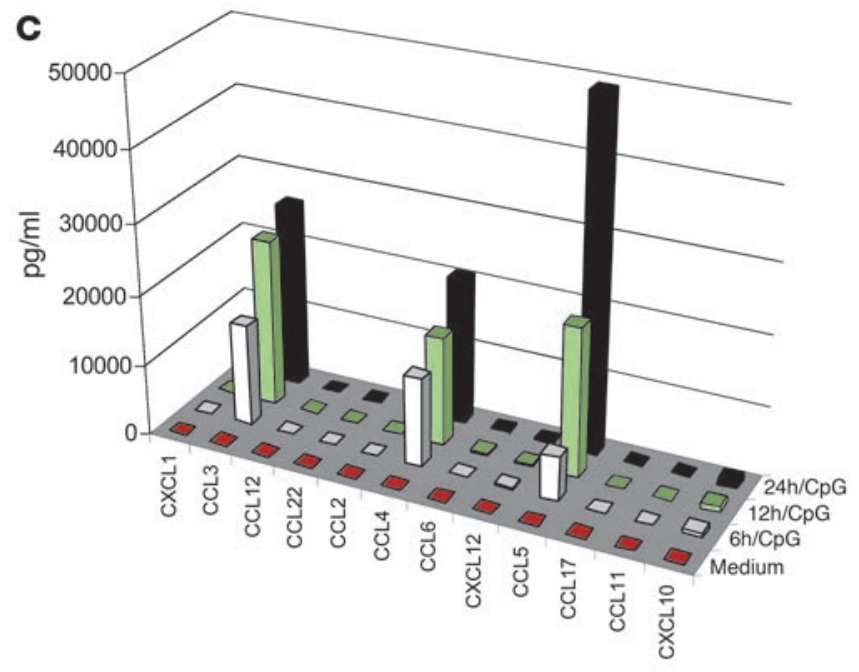

D

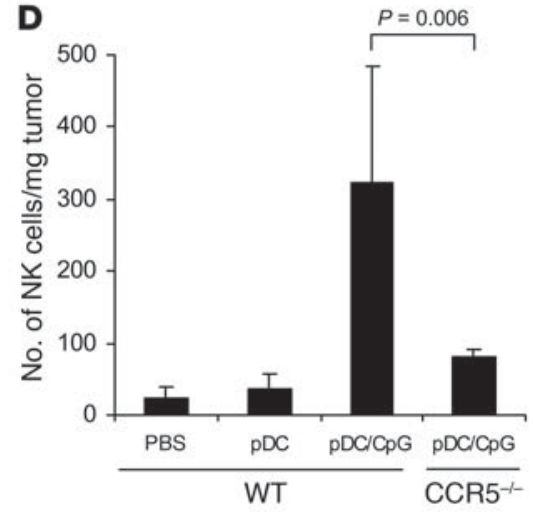

\section{Figure 5}

CCR5 is critical for pDCinduced NK cell migration into tumors. (A) Percentage of tumor-infiltrating NK cells at treatment day 2. (B) Microarray analysis of chemokine transcript expression in resting or CpG-activated pDCs. Signal intensities were normalized to the mean intensity of all the genes represented on the array. (C) Time course analysis of chemokine protein expression in pDCs following activation with $\mathrm{CpG}$. (D) Quantitation of NK cell tumor infiltration 2 days following pDC injection of wild-type or CCR5-deficient mice. Data are expressed as mean $\pm S D$ and are representative of 3 experiments with similar results.
IFNs produced by activated $\mathrm{pDCs}$ are responsible for the observed enhanced NK cell cytotoxicity.

OX40-OX40L interactions promote cell contact-dependent induction of IFN- $\gamma$ secretion by NK cells. We next investigated the mechanisms by which activated pDCs augment another major NK cell effector function, inflammatory cytokine secretion. Freshly isolated NK cells were cocultured with pDCs in the presence or absence of $\mathrm{CPG}$ activation, and IFN- $\gamma$ secretion was assessed by ELISA. As shown in Supplemental Figure 5A, in the presence of activated pDCs, NK cells produced much higher levels of IFN- $\gamma$ compared with NK cells alone plus CpG, or NK cells cocultured with resting pDCs. Intracellular flow cytometry confirmed that the IFN- $\gamma$ was produced by NK cells rather than by activated pDCs (data not shown).

To further define the mechanism by which activated pDCs induced IFN- $\gamma$ release by NK cells, the coculture experiment was repeated in the presence of a panel of neutralizing Abs. As shown in Supplemental Figure 5A, blocking IFN- $\alpha / \beta$ and IL-12 only partially blocked the ability of activated pDCs to induce IFN- $\gamma$ production by NK cells. Interestingly, separation of NK cells and activated pDCs by a transwell membrane even more strikingly inhibited this ability, suggesting that pDCs use additional cell contact-dependent mechanisms to elicit IFN- $\gamma$ production by NK cells (Supplemental Figure 5A). Blocking experiments using 

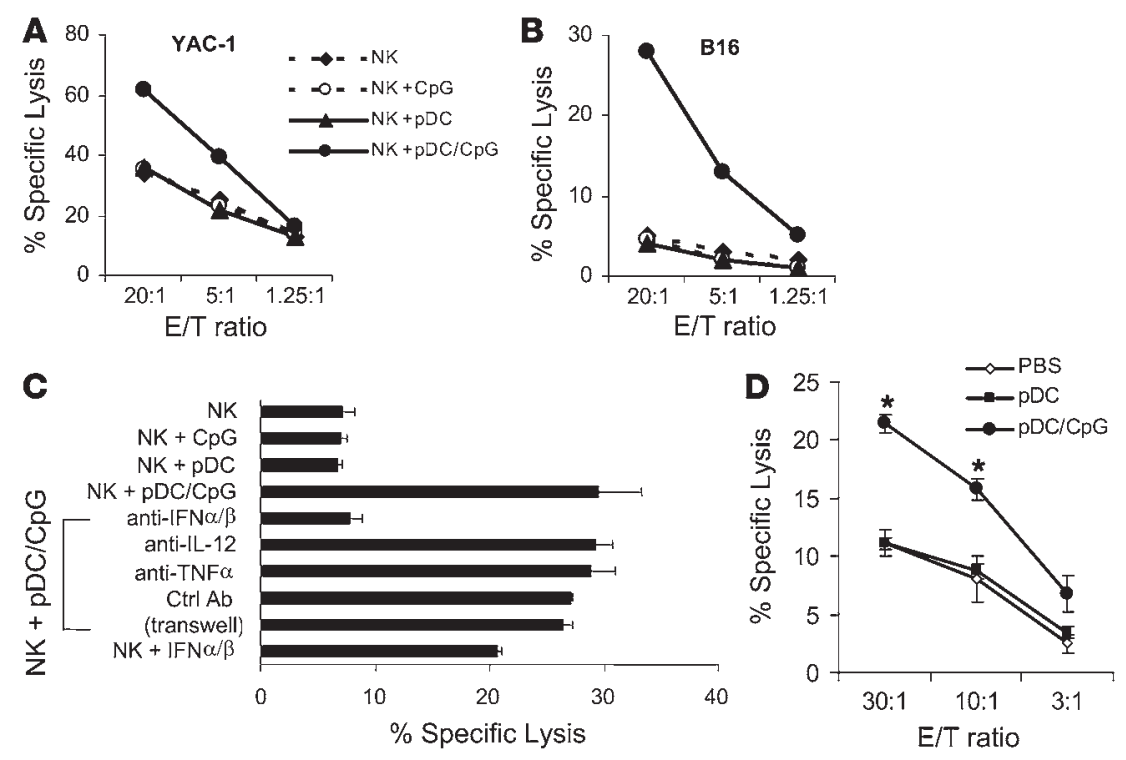

\section{Figure 6}

pDCs enhance NK cell cytolytic activity through secretion of type I IFNs. Sorted splenic NK cells were cocultured for $4 \mathrm{~h}$ with ${ }^{51} \mathrm{Cr}$-labeled YAC-1 (A) or B16 (B) tumor cells in the presence of CpG oligonucleotides, resting pDCs, CpG-activated $\mathrm{pDCs}$, or media alone. Specific chromium release was then assessed at different effector/ target cell ratios, as shown. (C) Similar experiment as in $\mathbf{B}$, except performed in the presence of blocking Abs, cytokines, or a transwell culture system with a 20:1 effector/target cell ratio, as indicated. (D) Mice were given intravenous pDC injection, and 2 days later NK cells were purified from spleen and assessed for cytotoxicity using B16 tumor cells as targets ( ${ }^{*} P=0.001$ for $\mathrm{pDC} / \mathrm{CpG}$ versus $\mathrm{pDC}$ ). Data are expressed as mean $\pm S D$ and are representative of 3 independent experiments with similar results.
mAbs against several candidate NK cell surface markers, including NK1.1, NKG2D, and CD244, demonstrated that these molecules were likely not involved.

To further explore the molecular mechanisms mediating the cell contact-dependent production of IFN- $\gamma$ by NK cells, we analyzed our microarray gene expression profile for candidate molecules specifically upregulated on the cell surface of $\mathrm{pDCs}$ upon $\mathrm{CpG}$ activation. In contrast to resting $\mathrm{pDCs}, \mathrm{CpG}$ stimulation induced $\mathrm{pDCs}$ to upregulate the expression of several members of the TNF superfamily, including OX40L, 4-1BBL, and TRAIL. We therefore screened these molecules for their ability to mediate cell contactdependent activation of NK cells by measuring IFN- $\gamma$ production using either neutralizing Abs or $\mathrm{pDC}$ from specific knockout mice. Among all the molecules assessed, OX40L appeared to be the most promising candidate, as its expression was significantly upregulated on activated $\mathrm{pDCs}$, and corresponding expression of OX40 was also detected on NK cells (Supplemental Figure 5B). Indeed, NK cells activated by pDCs from OX40L $/$ - mice showed significantly impaired IFN- $\gamma$ production compared with NK cells activated by wild-type pDCs (Supplemental Figure 5C). OX40L-deficient pDCs were found to secrete similar levels of cytokines IL-12, TNF- $\alpha$, and IFN- $\alpha$ to those produced by wild-type pDCs in response to CpG activation (data not shown). Combined blockade of interactions with the use of OX40L-deficient pDCs and IFN- $\alpha / \beta$-neutralizing Abs resulted in an even greater reduction in IFN- $\gamma$ production by NK cells, approximately 5 -fold less than that elicited by wild-type pDCs. This additive effect suggests that pDCs may promote NK cell cytokine secretion through distinct activation pathways.

Perforin and IFN- $\gamma$ play critical roles in pDC-mediated, NK cell-dependent $T$ cell cross-priming. To further address the molecular mechanisms behind the pDC-mediated antitumor immune response, we next asked whether perforin or IFN- $\gamma$ were important for NK cell-dependent CTL cross-priming in our model. Activated pDC treatment was therefore given to IFN- $\gamma^{-/-}$or perforin ${ }^{-/-}$mice bearing B16-OVA tumors, and 5 days following pDC injection the percentage of OVA-tetramer-positive $\mathrm{CD} 8^{+} \mathrm{T}$ cells was evaluated in the spleen. As shown in Figure 7, T cell cross-priming of OVAspecific $\mathrm{T}$ cells was completely abrogated in both perforin ${ }^{-/-}$mice and IFN- $\gamma^{-/-}$mice. Collectively, these results suggest that perforin and IFN- $\gamma$, likely produced by NK cells, both play critical roles in pDC-mediated, NK cell-dependent $\mathrm{T}$ cell cross-priming.

\section{Discussion}

The immune system is largely successful at controlling or eliminating viral infections but is comparatively ineffective at preventing the outgrowth of tumors or eliminating metastatic deposits. A critical difference between antiviral immune responses and antitumor responses is the recognition of PAMPs by innate immune cells through TLRs. TLR-activated pDCs at sites of viral infection serve to activate multiple other immune cell subsets and to initiate the adaptive immune responses that follow $(17,18)$. Several groups have reported that activated pDCs can strongly enhance the ability of cDCs to initiate antiviral CTL responses, but it has remained unclear whether $\mathrm{pDCs}$ can elicit effective antitumor

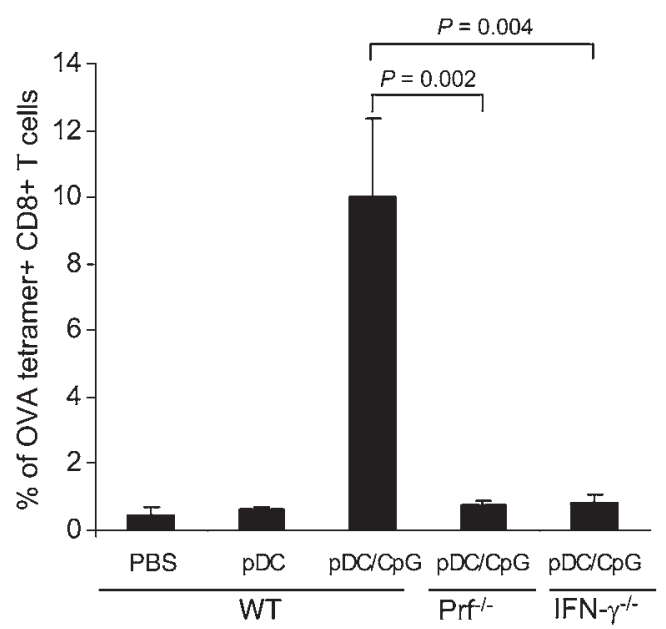

Figure 7

Both perforin and IFN- $\gamma$ are important for pDC-mediated CTL response. OVA-tetramer staining of splenocytes derived from pDC-treated B16OVA tumor-bearing wild-type, perforin-deficient ( $\mathrm{Prf}^{-/}$), or IFN- $\gamma-$ deficient mice. Data shown are expressed as mean \pm SEM and are representative of 3 independent experiments with similar results. 


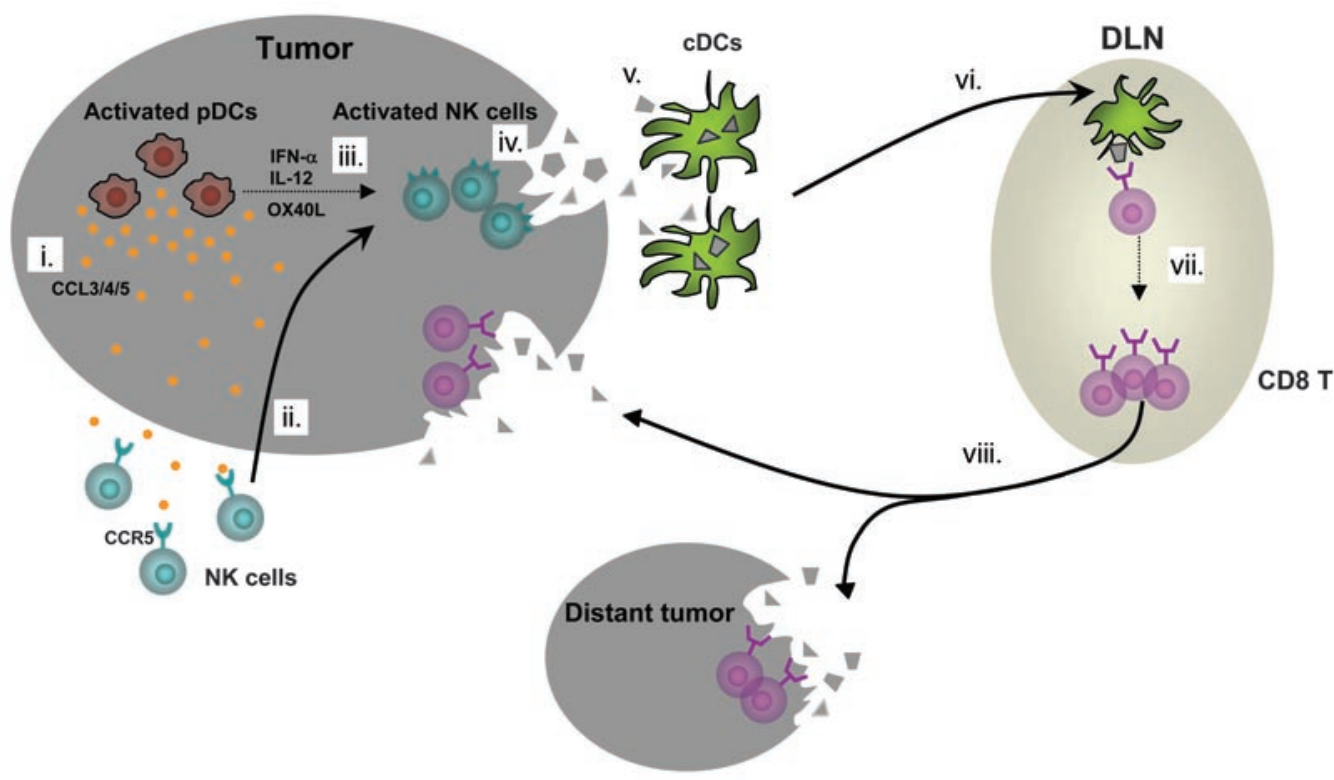

Figure 8

Immune cascade initiated by activated pDCs, culminating in a strong and systemic antitumor immune response. (i) CpG-activated pDCs produce large amounts of chemokines CCL3, CCL4, and CCL5 within the tumor microenvironment. (ii) NK cells migrate to tumor sites through CCR5-mediated chemotaxis. (iii) Recruited NK cells are activated to produce IFN- $\gamma$ by pDCs through cytokines and cell-to-cell interactions such as OX40/OX40L. (iv) Activated NK cells initiate tumor cell killing via enhanced cytolytic activity. (v) Tumor-associated antigens released by NK-mediated tumor destruction are taken up by endogenous cDCs, which then (vi) become activated and migrate to tumor DLN. (vii) Cross-presentation of tumor antigens by activated cDCs in DLN leads to effective cross-priming and expansion of tumor antigen-specific T cells. (viii) Infiltration of both treated and untreated tumors by antigen-specific T cells mediates further tumor cell killing and systemic antitumor immunity.

responses by facilitating spontaneous $\mathrm{T}$ cell cross-priming against tumor-associated antigens.

This study sought to address this question using an in vivo tumor model. Our data reveal that activated pDCs can indeed orchestrate specific and systemic adaptive antitumor immune responses against B16 tumors and do so by inducing the rapid recruitment and activation of NK cells into the tumor microenvironment that, in turn, leads to subsequent cross-priming of tumor antigen-specific CTLs (Figure 8).

While pDC-NK cell interactions in vivo remain largely uncharacterized, crosstalk between cDCs and NK cells has been well documented (19-21). NK cell activation by cDCs is known to involve signals from both soluble cytokines and direct cell-to-cell contact (21-26). While this also appears to true for pDC-mediated NK cell activation, the molecular interactions mediating this effect are quite different. In contrast to cDCs, pDC-mediated induction of NK cell cytolytic activity appeared to be mostly dependent on soluble Type I IFNs, with cell-to-cell contact crucial for inducing maximal NK cell cytokine production. Although NKG2D-NKG2D ligand interactions are important for NK cell activation by cDCs (26), this molecular interaction did not seem to be involved in pDC-mediated NK cell activation. By contrast, OX40-OX40L interactions appeared to play a significant role in the induction of IFN- $\gamma$ secretion from NK cells by activated pDCs. However, since blocking OX40-OX40L interactions did not completely abrogate IFN- $\gamma$ secretion, additional as yet undefined mechanisms are likely to be involved.
Consistent with previous studies, we found that $\mathrm{pDCs}$ produce large amounts of chemokines CCL3, CCL4, and CCL5 upon stimulation with CpG (Figure 5) $(4,27)$. NK cell recruitment to $\mathrm{pDC}$ injected sites is likely to be mediated through those chemokines interacting with CCR5, as NK cells from $\mathrm{CCR}^{-/-}$mice showed a severe impairment in the ability to migrate to tumors injected with activated pDCs. Within the tumor microenvironment, activated NK cells may also secrete CCL3, CCL4, and CCL5, therefore constituting a positive loop to recruit more NK cells (24). Interestingly, chemokines CCL3, CCL4, and CCL5 are also known to induce the migration of activated memory $\mathrm{T}$ cells as well as monocytes $(28,29)$. The potential role of those cells in the generation of pDC-mediated antitumor immunity will require further characterization.

Two to three days following the recruitment of $\mathrm{NK}$ cells into the tumor microenvironment, cross-priming of tumor antigen-specific $\mathrm{CD}^{+} \mathrm{T}$ cells could be detected in tumor DLN. Notably, this was exquisitely dependent upon NK cells, as NK cell depletion completely abrogated cross-priming (Figure 4), as did removal of perforin and IFN- $\gamma$ (Figure 7). Activated NK cells may facilitate the generation of adaptive $\mathrm{T}$ cell-mediated antitumor immunity through several potential mechanisms: (a) NK cell-induced tumor lysis could liberate tumor antigens that are subsequently taken up by cDCs, processed and cross-presented in order to prime antigen-specific CTLs. (b) NK cells can regulate cDC activation by enhancing their ability to produce proinflammatory cytokines and to elicit tumor-specific $\mathrm{T}$ cell responses (30-33). (c) IFN- $\gamma$ upregulates MHC class I and class II expression in many tissues, including tumor cells and cDCs, possibly enhancing both direct and cross-presentation of tumor antigens (34). (d) IFN- $\gamma$ produced by NK cells may directly stimulate CTL generation and helper $\mathrm{T}$ cell responses (35).

Although it has been shown that pDCs are capable of cross-presenting exogenous antigens in vitro, it remains an open question whether pDCs are able to effectively cross-prime $T$ cells in vivo (14, 36). Our data suggest that endogenous cDCs, not pDCs, migrating from the tumor site to tumor DLN likely act as the crucial intermediate APC type, bridging the gap between NK cell recruitment to the tumor site and T cell cross-priming in the tumor DLN (Figure 8). This is supported by several observations: (a) Only CD $11 \mathrm{c}^{+}$ cDCs isolated from B16-OVA-GFP tumor DLN following activated $\mathrm{pDC}$ injection carried antigen from the tumor site. (b) cDCs, but 
not $\mathrm{pDCs}$, purified from tumor DLN were capable of stimulating tumor antigen-specific T cells ex vivo. (c) Increased numbers of activated cDCs were found in activated $\mathrm{pDC}$-treated tumor DLN compared with non-draining LNs or tumor DLN from mice injected with resting pDCs. In addition, treatment of B16-OVA tumor-bearing mice with pDCs derived from bm 1 transgenic mice, which are incapable of presenting the $\mathrm{H} 2-\mathrm{K}^{\mathrm{b}}$-restricted $\mathrm{OVA}_{257-264}$ peptide, showed similar levels of OVA-specific T cell cross-priming compared with those treated with pDC from wild-type mice, excluding the possibility that injected pDCs cross-presented tumor antigens in our model (Supplemental Figure 6). These observations support the findings that pDCs are less efficient than $\mathrm{cDCs}$ in vivo at cross-priming $\mathrm{T}$ cells against exogenous antigens (15).

In addition to responding to NK cell-derived signals, cDCs can also be strongly activated by pDCs. We and others have previously shown that activated pDCs can enhance the ability of cDCs to prime a CTL response $(12,37,38)$. Both direct cell-to-cell contact and type I IFNs have been demonstrated to be important in promoting generation of $\mathrm{CD}^{+} \mathrm{T}$ cell responses mediated by $\mathrm{cDCs}$. IFN- $\alpha$ has been shown as a potent licensor of $\mathrm{CDC}$-mediated T cell crosspriming, possibly through upregulating the expression of $\mathrm{MHC}$ class I costimulatory molecules, TAP-1, and TLRs on cDCs as well as enhancing cell viability (39-41). Thus, it is likely that a combination of signals from $\mathrm{pDCs}$, in conjunction with help from recruited NK cells, contributes to the licensing of cDCs to cross-prime the robust antigen-specific CTL response seen in our model.

These results suggest that harnessing the use of pDCs in cancer immunotherapy may represent a nonspecific innate immune manipulation that can ultimately manifest as a specific, adaptive antitumor immune response. In this context, it is important to note that the $\mathrm{CD}^{+} \mathrm{T}$ cell response induced by activated $\mathrm{pDC}$ injection was directed against multiple tumor-associated antigens, as we detected relatively robust CTL responses not only against a neo-antigen, OVA, but also against an endogenous self antigen, p15E. Equally important in the metastatic clinical setting, the CTL response generated by a single i.t. injection of pDCs was systemic in nature, infiltrating and slowing the growth of uninjected contralateral tumors. Although the antitumor activity at the distant tumors was relatively modest compared with injected tumors, the significant infiltration of distant tumors by tumor antigen-specific $T$ cells suggests that activated $\mathrm{pDCs}$ have promising therapeutic potential for use in cancer treatments. It is also of interest that injection of activated pDCs in combination with CD4 depletion induced even more dramatic antitumor activity. This may indicate that $\mathrm{CD}^{+}$regulatory $\mathrm{T}$ cells are involved in suppressing the $\mathrm{pDC}$ mediated antitumor immune response, although further investigation will be required to confirm this.

Clinically, the use of ex vivo-generated pDCs would present a formidable challenge, as large-scale human pDC generation and isolation is not currently feasible. However, pDCs have been found to reside in several human tumors and are therefore potentially ideal targets for activation with TLR7 and TLR9 agonists (42-45). Such therapeutic interventions have met with success in mouse tumor models, and several cancer trials are also currently underway to test the efficacy of these agents in humans (46-48). In this context, critical questions will be whether $\mathrm{pDC}$ numbers in situ are sufficient to initiate an effective antitumor response and whether their activation potential is impaired by an immunosuppressive tumor microenvironment. It is also important to note that under certain circumstances, pDCs have been shown to suppress immune responses and are a negative prognostic factor in cancers such as primary breast cancer (49). However, the majority of these tumor-infiltrating pDCs were found to be either immature in phenotype or functionally impaired. It is thus possible that, similar to cDCs, resting and activated $\mathrm{pDCs}$ may have opposite functions that can lead either to suppression or induction of immune responses, respectively.

Gaining insights into pDC-mediated mechanisms of immune orchestration will be critical for designing the next generation of cancer immunotherapeutics, especially if TLR agonists themselves prove insufficient to halt tumor growth. For example, our study suggests that ectopic expression of CCR5 ligands at the tumor site could lead to NK cell infiltration, with exogenously provided IFN- $\alpha$ (currently available for clinical use) providing recruited NK cells with an activation signal enhancing their lytic function. Our results imply the possibility that such nonspecific interventions could ultimately set the stage for the generation of a broad adaptive antitumor immune response of multiple specificities.

\section{Methods}

Mice. Female C57BL/6, Ccr5-, Prf-, IFN- $\gamma$-, and OX40L-deficient mice and bm 1 mice (C57BL/ 6 background) were purchased from the NCI, Taconic, or The Jackson Laboratory and maintained in a pathogen-free animal facility at the MD Anderson Cancer Center. Mice were used at 8-10 weeks of age, and all animal experiments were performed according to protocols approved by the Institutional Animal Care and Use Committee at The University of Texas MD Anderson Cancer Center.

Cell lines and reagents. B16, B16-OVA, B16-OVA-GFP, YAC-1 and MCA205 tumor lines were cultured in RPMI 1640 supplemented with $10 \%$ heatinactivated FBS, L-glutamine, sodium pyruvate, nonessential amino acids, and penicillin-streptomycin (all from Invitrogen/Life Technologies Inc.). Anti-mouse monoclonal Abs against CD4 (GK1.5) and CD8 (2.43) were obtained from the National Cancer Institute Biological Resources Branch Preclinical Repository. Anti-NK1.1 Ab was made from PK136 hybridoma cells (ATCC). Anti-asialo GM1 Ab against mouse NK cells was purchased from Wako Pure Chemical Industries. Abs used for flow cytometry analysis and cell sorting were purchased from $\mathrm{BD}$ - Pharmingen unless otherwise indicated. Neutralizing Abs against mouse IFN- $\alpha$ and IFN- $\beta$ were purchased from PBL Biomedical Laboratories. Neutralizing Abs against mouse TNF- $\alpha$ and IL-12 were purchased from R\&D Systems. CPG-ODN2216 was synthesized by Invitrogen Life Technologies.

pDC and NK cell isolation. Murine pDCs and NK cells were isolated from the bone marrow and spleen, respectively, of Flt3L-treated mice. An expression vector encoding full-length murine Flt $3 \mathrm{~L} c \mathrm{cNA}$ (pORF-mFlt3L) was purchased from Invivogen. Injection of $10 \mu \mathrm{g}$ of plasmid DNA encoding Flt3L was performed using the hydrodynamic-based gene delivery technique, as previously described (50). Bone marrow cells were isolated from femurs and tibiae 10 days after Flt3L plasmid injection, incubated with rat anti-CD16/32 mAbs (2.4G2) to block nonspecific binding, and then stained with the following mAbs: anti-CD11c, anti-B220, and anti-CD11b (all from BD Biosciences). pDCs with a CD11 $\mathrm{c}^{\text {int } C D 11 b-B 220^{+}}$phenotype were sorted using a BD FACSAria. Although pDCs from Flt3L-treated mice have been shown to demonstrate a slightly more activated phenotype compared with pDCs from saline-treated mice, no significant phenotypic differences were observed in these 2 populations following CpG activation (data not shown). NK cells were isolated using DX5 microbeads (Miltenyi Biotec) and then sorted for the NK1.1 $1^{+} \mathrm{CD}^{-}$cell population. Cell purity was greater than $98 \%$ after sorting. pDCs were stimulated with or without CpG-ODN-2216 $(10 \mu \mathrm{g} / \mathrm{ml})$ for $6 \mathrm{~h}$ for in vivo transfer experiments or overnight for in vitro studies. Cells were extensively washed at least 3 times with PBS before in vivo transfer. 
Tumor treatment and monitoring. C57BL/6 mice (5-10 mice per group) were subcutaneously inoculated with $3-5 \times 10^{5} \mathrm{~B} 16$ tumor cells on day -7 or day -8 . Tumor-bearing mice were treated on day 0 with i.t. injection of $2 \times 10^{6}$ resting or $6 \mathrm{~h} \mathrm{CpG-activated} \mathrm{pDCs.} \mathrm{Tumor} \mathrm{growth} \mathrm{and} \mathrm{mouse} \mathrm{survival} \mathrm{were}$ monitored. To study whether i.t. injection of pDCs was able to induce systemic antitumor activity, mice were subcutaneously inoculated with $3 \times 10^{5}$ B16 on left flank on day -7 and $2 \times 10^{5}$ B16 on right flank on day -2 . Mice were treated with resting or activated pDCs by i.t. injection in tumors of left flank. CD4 and CD8 T cell depletions were performed by using anti-mouse CD4 (GK1.5) or CD8 (2.43) specific mAbs, intravenously injected (200 $\mu \mathrm{g} /$ mouse) on days -2 and 0 . Intraperitoneal injections were repeated every 6 days thereafter during the experiment to maintain CD4 and CD8 cell depletion. In some experiments, NK cells were depleted by intraperitoneal injection of rabbit anti-asialo GM1 $(200 \mu \mathrm{l})$ on days $-2,0$, and 2 or depleted by intraperitoneal injection of anti-NK1.1 $\mathrm{mAb}(100 \mu \mathrm{g})$ on days $-2,-1,0$, and 2 . Tumor growth was monitored by measuring the perpendicular diameters of tumors. Mice were sacrificed when tumor size reached $20 \mathrm{~mm}$ in diameter.

Immunohistochemistry. Immunohistochemistry was performed on frozen sections of mouse tumor from mice receiving either control or activated pDCs. Abs to NK cells (anti-Ly-49G2, clone 4D11) and CD8 T cells (CD8 $\alpha, \mathrm{CD} 3)$ were used.

Tumor antigen-specific T cell response. pDCs were purified and stimulated with or without $\mathrm{CPG}$ for $6 \mathrm{~h}$ as described above, then washed with PBS before in vivo transfer. B16-OVA or B16 tumor-bearing mice were sacrificed 5 days after i.t. injection of pDCs. Splenocytes were harvested, and erythrocytes were depleted using ACK lysing buffer. Cells were stimulated with or without $1 \mu \mathrm{M} \mathrm{OVA}_{257-264}$ (SIINFEKL) or p15E peptide (KSPWFTTL) in the presence of Brefeldin A for $6 \mathrm{~h}$. Cells were collected and incubated with rat anti-CD16/32 mAbs (2.4G2) to block nonspecific binding and then stained with anti-CD8 $\alpha$ and intracellular IFN- $\gamma$ according to the manufacturer's instructions (BD Biosciences). If indicated, NK cells were depleted by anti-asialo GM1 or anti-NK1.1 Ab. To quantitate tumor antigen specificity of tumor-infiltrating $\mathrm{T}$ cells, B16-OVA tumor-bearing mice were sacrificed 5 days following pDC injection. Single-cell suspensions were prepared from tumor tissues and stained with anti-CD8 $\alpha$ and OVAtetramer, with dead cells excluded by PI staining. In some experiments, $\mathrm{Prf}^{-/-}$mice or IFN- $\gamma^{-/-}$mice received $\mathrm{pDC}$ injection for established B16-OVA tumors. As above, antigen-specific $\mathrm{T}$ cell responses were evaluated using OVA tetramer staining 5 days later.

DC preparation and cross-priming assay. B16-OVA or B16-OVA-GFP tumorbearing mice were sacrificed 16 to $32 \mathrm{~h}$ after PDC injection, and DLN and non-DLN were harvested for preparing single-cell suspensions. The absolute numbers and percentages of $\mathrm{cDCs}\left(\mathrm{CD} 11 \mathrm{c}^{+} \mathrm{B} 220^{-} \mathrm{CD} 3^{-} \mathrm{CD} 19^{-} \mathrm{DX} 5^{-}\right)$ and $\mathrm{pDCs}\left(\mathrm{CD} 11 \mathrm{c}^{\mathrm{int}} \mathrm{B} 220^{+} \mathrm{CD} 3^{-} \mathrm{CD} 19^{-} \mathrm{DX} 5^{-}\right)$, levels of CD86 expression, and percentages of $\mathrm{GFP}^{+}$DCs were determined by flow cytometry. Dead cells were excluded by PI staining. To assess the ability of DC subsets to cross-prime OVA-specific T cells, cDC and pDC subsets were purified from tumor DLN from pooled mice ( 5 to 6 mice per group) by cell sorting and then analyzed ex vivo by coculture with activated OT-I T cells for $12 \mathrm{~h}$ at a 1:1 ratio. Activated OT-I T cells were prepared by culturing splenocytes derived from OT-I mice for 6 days in the presence of IL-2 and OVA $257-264$ peptide. T cell activation was evaluated using intracellular IFN- $\gamma$ staining.

Assessment of NK cell migration. The migration of endogenous NK cells in response to $\mathrm{pDC}$ injection was evaluated in B16 tumor-bearing mice. Mice were i.t. injected with resting or activated $\mathrm{pDCs}$ and sacrificed on day 2 . Single-cell suspensions were prepared from tumors and stained with NK1.1-, CD3-, CD45-, and CD69-specific mAbs. Non-viable cells were excluded by $\mathrm{PI}$ staining. CCR $5^{-/}$mice were used as recipients in some experiments.

Microarray analysis and bioinformatics. pDCs were sorted as described above to a purity of greater than $99 \%$. Total RNA from resting or CpG-activated pDCs was immediately isolated using an RNeasy kit (Qiagen) and used to generate cDNA and cRNA to hybridize onto the mouse Genome 4302.0 array, according to the manufacturer's protocol (Affymetrix). Scanned images were analyzed by the Affymetrix GeneChip software Microarray Suite 5.0. Signal intensities were normalized to the mean intensity of all the genes represented on the array, and global scaling was applied before comparison analysis.

NK cell cytotoxicity assay. pDCs were activated overnight with or without CpG. NK cells (at a DC/NK ratio of 1:1) and ${ }^{51} \mathrm{Cr}$-labeled tumor cell lines B16 or YAC- 1 cells at $1 \times 10^{4}$ target cells/well at different E:T ratios were added into the culture as triplicates in round-bottom 96-well plates. After $4 \mathrm{~h},{ }^{51} \mathrm{Cr}$ release was determined against target cells. Specific ${ }^{51} \mathrm{Cr}$ release was calculated as a percentage using the standard formula: ([sample release - spontaneous release]/[total release - spontaneous release] $) \times 100$. Neutralizing Abs against mouse IFN- $\alpha\left(1 \times 10^{4} \mathrm{U} / \mathrm{ml}\right), \operatorname{IFN}-\beta\left(1 \times 10^{4}\right.$ $\mathrm{U} / \mathrm{ml}), \mathrm{TNF}-\alpha(4.8 \mu \mathrm{g} / \mathrm{ml})$, and IL-12 $(6.7 \mu \mathrm{g} / \mathrm{ml})$ were added at the beginning of culture. Transwell studies were performed by plating pDCs in the upper chamber and NK cells in the lower chamber of a $0.4-\mu \mathrm{m}$ transwell plate. For testing NK activation by pDCs in vivo, mice were intravenously injected with $\mathrm{CpG}$-activated or resting pDCs. Mice were sacrificed $36 \mathrm{~h}$ after $\mathrm{pDC}$ injection, and NK cells were isolated from mouse spleens using DX5 microbeads. The cytotoxic activity of NK cells was analyzed using ${ }^{51} \mathrm{Cr}$-labeled B16 tumor cells at a $1 \times 10^{4}$ target cells/well at different E:T ratios as triplicates in roundbottom 96 -well plates. Specific ${ }^{51} \mathrm{Cr}$ release was calculated as above.

Cytokine ELISA and flow cytometry. NK and pDCs were purified as described above. pDCs were cocultured with NK cells at a $\mathrm{pDC} / \mathrm{NK}$ cell ratio of $1: 1$ $\left(1 \times 10^{5} \mathrm{each}\right)$ in the presence or absence of $\mathrm{CPG}$. IFN- $\gamma$ production was determined in supernatant after $20 \mathrm{~h}$ of coculture using ELISA kits (Pierce Biotechnology Inc.). Neutralizing Abs and transwell studies were performed as described above. The levels of chemokine produced by pDCs at different time points after $\mathrm{CpG}$ stimulation were performed using either searchlight array (Pierce Biotechnology Inc.) or ELISA (R\&D Systems). The expression of OX40L and OX40 on pDC and NK cells was also tested using flow cytometry.

Statistics. The statistical analyses to compare tumor size, the percentage and absolute number of cells, cytolytic activity, and cytokine levels were determined using the Mann-Whitney nonparametric $U$ test or Student's $t$ test. The statistical analysis to compare survival was determined using Kaplan-Meier test.

\section{Acknowledgments}

This work was supported by NIH grant R01 CA123182-02 (to P. Hwu).

Received for publication August 13, 2007, and accepted in revised form December 12, 2007.

Address correspondence to: Patrick Hwu, Department of Melanoma Medical Oncology, Center for Cancer Immunology Research, University of Texas MD Anderson Cancer Center, 1515 Holcombe Blvd., Houston, Texas 77030, USA. Phone: (713) 563-1727; Fax: (713) 745-1046; E-mail: phwu@mdanderson.org. Or to: Gang Wang, US Food and Drug Administration, Center for Biologics Evaluation and Research, 1401 Rockville Pike, HFM-675, Rockville, MD 20852, USA. Phone: (301) 827-7123; Fax: (301) 827-3536; E-mail: gang.wang@fda.hhs.gov.

Gang Wang's present address is: U.S. Food and Drug Administration, Center for Biologics Evaluation and Research, Rockville, Maryland, USA.

Chengwen Liu and Yanyan Lou contributed equally to this work. 
1. Fearon, D.T., and Locksley, R.M. 1996. The instructive role of innate immunity in the acquired immune response. Science. 272:50-54.

2. Medzhitov, R., and Janeway, C.A., Jr. 1997. Innate immunity: the virtues of a nonclonal system of recognition. Cell. 91:295-298.

3. McKenna, K., Beignon, A.S., and Bhardwaj, N. 2005. Plasmacytoid dendritic cells: linking innate and adaptive immunity. J. Virol. 79:17-27.

4. Megjugorac, N.J., et al. 2004. Virally stimulated plasmacytoid dendritic cells produce chemokines and induce migration of T and NK cells. J. Leukoc. Biol. 75:504-514.

5. Dalod, M., et al. 2003. Dendritic cell responses to early murine cytomegalovirus infection: subset functional specialization and differential regulation by interferon alpha/beta. J. Exp. Med. 197:885-898.

6. Jego, G., et al. 2003. Plasmacytoid dendritic cells induce plasma cell differentiation through type I interferon and interleukin 6. Immunity 19:225-234.

7. Gerosa, F., et al. 2002. Reciprocal activating interaction between natural killer cells and dendritic cells. J. Exp. Med. 195:327-333.

8. Marrack, P., Kappler, J., and Mitchell, T. 1999. Type I interferons keep activated T cells alive. J. Exp. Med. 189:521-530.

9. Barchet, W., Cella, M., and Colonna, M. 2005. Plasmacytoid dendritic cells - virus experts of innate immunity. Semin. Immunol. 17:253-261.

10. Blanco, P., Palucka, A.K., Gill, M., Pascual, V., and Banchereau, J. 2001. Induction of dendritic cell differentiation by IFN-alpha in systemic lupus erythematosus. Science. 294:1540-1543.

11. Nestle, F.O., et al. 2005. Plasmacytoid predendritic cells initiate psoriasis through interferon-alpha production. J. Exp. Med. 202:135-143.

12. Lou, Y., et al. 2007. Plasmacytoid dendritic cells synergize with myeloid dendritic cells in the induction of antigen-specific antitumor immune responses. J. Immunol. 178:1534-1541.

13. Zeh, H.J., 3rd, Perry-Lalley, D., Dudley, M.E., Rosenberg, S.A., and Yang, J.C. 1999. High avidity CTLs for two self-antigens demonstrate superior in vitro and in vivo antitumor efficacy. J. Immunol. 162:989-994.

14. Shinohara, M.L., et al. 2006. Osteopontin expression is essential for interferon-alpha production by plasmacytoid dendritic cells. Nat. Immunol. 7:498-506

15. Salio, M., Palmowski, M.J., Atzberger, A., Hermans, I.F., and Cerundolo, V. 2004. CpG-matured murine plasmacytoid dendritic cells are capable of in vivo priming of functional CD8 $\mathrm{T}$ cell responses to endogenous but not exogenous antigens. J. Exp. Med. 199:567-579.

16. Biron, C.A., Nguyen, K.B., Pien, G.C., Cousens, L.P., and Salazar-Mather, T.P. 1999. Natural killer cells in antiviral defense: function and regulation by innate cytokines. Annu. Rev. Immunol. 17:189-220.

17. Liu, Y.J. 2005. IPC: professional type 1 interferonproducing cells and plasmacytoid dendritic cell precursors. Annu. Rev. Immunol. 23:275-306.

18. Colonna, M., Trinchieri, G., and Liu, Y.J. 2004. Plasmacytoid dendritic cells in immunity. Nat. Immunol. 5:1219-1226.

19. Degli-Esposti, M.A., and Smyth, M.J. 2005. Close encounters of different kinds: dendritic cells and NK cells take centre stage. Nat. Rev. Immunol. 5:112-124.

20. Wallace, M.E., and Smyth, M.J. 2005. The role of natural killer cells in tumor control - effectors and regulators of adaptive immunity. Springer Semin. Immunopathol. 27:49-64.

21. Moretta, A. 2005. The dialogue between human natural killer cells and dendritic cells. Curr. Opin. Immunol. 17:306-311.

22. Marcenaro, E., Ferranti, B., and Moretta, A. 2005. NK-DC interaction: on the usefulness of autoaggression. Autoimmun. Rev. 4:520-525.

23. Walzer, T., Dalod, M., Robbins, S.H., Zitvogel, L., and Vivier, E. 2005. Natural-killer cells and dendritic cells: "l'union fait la force". Blood. 106:2252-2258.

24. Walzer, T., Dalod, M., Vivier, E., and Zitvogel, L. 2005. Natural killer cell-dendritic cell crosstalk in the initiation of immune responses. Expert Opin. Biol. Ther. 5:S49-S59.

25. Koka, R., et al. 2004. Cutting edge: murine dendritic cells require IL-15R alpha to prime NK cells. J. Immunol. 173:3594-3598.

26. Andoniou, C.E., et al. 2005. Interaction between conventional dendritic cells and natural killer cells is integral to the activation of effective antiviral immunity. Nat. Immunol. 6:1011-1019.

27. Piqueras, B., et al. 2006. Upon viral exposure, myeloid and plasmacytoid dendritic cells produce 3 waves of distinct chemokines to recruit immune effectors. Blood. 107:2613-2618.

28. Cook, D.N., et al. 1995. Requirement of MIP-1 alpha for an inflammatory response to viral infection. Science. 269:1583-1585.

29. Sallusto, F., Lenig, D., Mackay, C.R., and Lanzavecchia, A. 1998. Flexible programs of chemokine receptor expression on human polarized $\mathrm{T}$ helper 1 and 2 lymphocytes. J. Exp. Med. 187:875-883.

30. Mocikat, R., et al. 2003. Natural killer cells activated by MHC class I(low) targets prime dendritic cells to induce protective CD8 T cell responses. Immunity. 19:561-569.

31. Kelly, J.M., et al. 2002. Induction of tumor-specific $\mathrm{T}$ cell memory by NK cell-mediated tumor rejection. Nat. Immunol. 3:83-90.

32. Westwood, J.A., et al. 2004. Cutting edge: novel priming of tumor-specific immunity by NKG2Dtriggered NK cell-mediated tumor rejection and Th1-independent CD4+ T cell pathway. J. Immunol. 172:757-761.

33. Dao, T., et al. 2005. Natural killer cells license dendritic cell cross-presentation of B lymphoma cell Associated antigens. Clin. Cancer Res. 11:8763-8772.

34. van den Elsen, P.J., Gobin, S.J., van Eggermond, M.C., and Peijnenburg, A. 1998. Regulation of MHC class I and II gene transcription: differences and similarities. Immunogenetics. 48:208-221.

35. Smyth, M.J., and Kelly, J.M. 1999. Accessory function for NK1.1+ natural killer cells producing interferon-gamma in xenospecific cytotoxic $\mathrm{T}$ lymphocyte differentiation. Transplantation. 68:840-843.

36. Haeryfar, S.M. 2005. The importance of being a $\mathrm{pDC}$ in antiviral immunity: the IFN mission versus Ag presentation? Trends Immunol. 26:311-317.

37. Le Bon, A., et al. 2003. Cross-priming of CD8+ T cells stimulated by virus-induced type I interferon. Nat. Immunol. 4:1009-1015.

38. Yoneyama, H., et al. 2005. Plasmacytoid DCs help lymph node DCs to induce anti-HSV CTLs. J. Exp. Med. 202:425-435.

39. Beignon, A.S., Skoberne, M., and Bhardwaj, N. 2003. Type I interferons promote cross-priming: more functions for old cytokines. Nat. Immunol. 4:939-941.

40. Le Bon, A., and Tough, D.F. 2002. Links between innate and adaptive immunity via type I interferon. Curr. Opin. Immunol. 14:432-436.

41. Cho, H.J., et al. 2002. IFN-alpha beta promote priming of antigen-specific CD8+ and CD4+ T lymphocytes by immunostimulatory DNA-based vaccines. J. Immunol. 168:4907-4913.

42. Zou, W., et al. 2001. Stromal-derived factor-1 in human tumors recruits and alters the function of plasmacytoid precursor dendritic cells. Nat. Med. 7:1339-1346

43. Mohty, M., Olive, D., and Gaugler, B. 2004. Plasmacytoid DCs and cancer: a new role for an enigmatic cell. Trends Immunol. 25:397-398; author reply 398-399.

44. Vermi, W., et al. 2003. Recruitment of immature plasmacytoid dendritic cells (plasmacytoid monocytes) and myeloid dendritic cells in primary cutaneous melanomas. J. Pathol. 200:255-268.

45. Hartmann, E., et al. 2003. Identification and functional analysis of tumor-infiltrating plasmacytoid dendritic cells in head and neck cancer. Cancer Res. 63:6478-6487.

46. Davila, E., and Celis, E. 2000. Repeated administration of cytosine-phosphorothiolated guanine-containing oligonucleotides together with peptide/ protein immunization results in enhanced CTL responses with anti-tumor activity. J. Immunol. 165:539-547.

47. Craft, N., et al. 2005. The TLR7 agonist imiquimod enhances the anti-melanoma effects of a recombinant listeria monocytogenes vaccine. J. Immunol. 175:1983-1990.

48. Molenkamp, B.G., et al. 2007. Intradermal CpG-B activates both plasmacytoid and myeloid dendritic cells in the sentinel lymph node of melanoma patients. Clin. Cancer Res. 13:2961-2969.

49. Treilleux, I., et al. 2004. Dendritic cell infiltration and prognosis of early stage breast cancer. Clin. Cancer Res. 10:7466-7474.

50. Wang, G., et al. 2003. In vivo antitumor activity of interleukin 21 mediated by natural killer cells. Cancer Res. 63:9016-9022. 